\title{
A 78-Year-Old Female with a Diffuse Pruritic Rash and Palmoplantar Desquamation
}

\author{
Gabriel E. Molina $^{a}$ Melissa J. Danesh ${ }^{b}$ d Ruth K. Foreman ${ }^{c}$ \\ Daniela Kroshinsky ${ }^{d}$ \\ ${ }^{a}$ Harvard Medical School, Boston, MA, USA; ${ }^{b}$ Harvard Combined Dermatology Residency \\ Program, Boston, MA, USA; ' Department of Pathology, Massachusetts General Hospital, \\ Boston, MA, USA; ${ }^{d}$ Department of Dermatology, Massachusetts General Hospital, Boston, \\ MA, USA
}

\section{Keywords}

Mycosis fungoides · Cutaneous T-cell lymphoma - Erythema annulare centrifugum

\section{Abstract}

Mycosis fungoides, the most common cutaneous T-cell lymphoma, is known to exhibit varied clinical presentations and mimic many other dermatoses. This morphological heterogeneity can often lead to initial misdiagnoses resulting in significant delays from symptom onset to diagnosis. We report a case of mycosis fungoides imitating clinical and histopathological features of erythema annulare centrifugum.

\section{Case Report}

A 78-year-old female with a history of chronic plaque psoriasis and hidradenitis suppurativa was admitted with hypertensive emergency and a 2-month history of a diffuse, pruritic rash and palmoplantar desquamation. She had had multiple outpatient dermatology visits in the preceding 2 months, including an initial visit 2 weeks after the rash's onset, at which time she was noted to have gyrate erythema as well as fine palmoplantar desquamation (Fig. 1). 

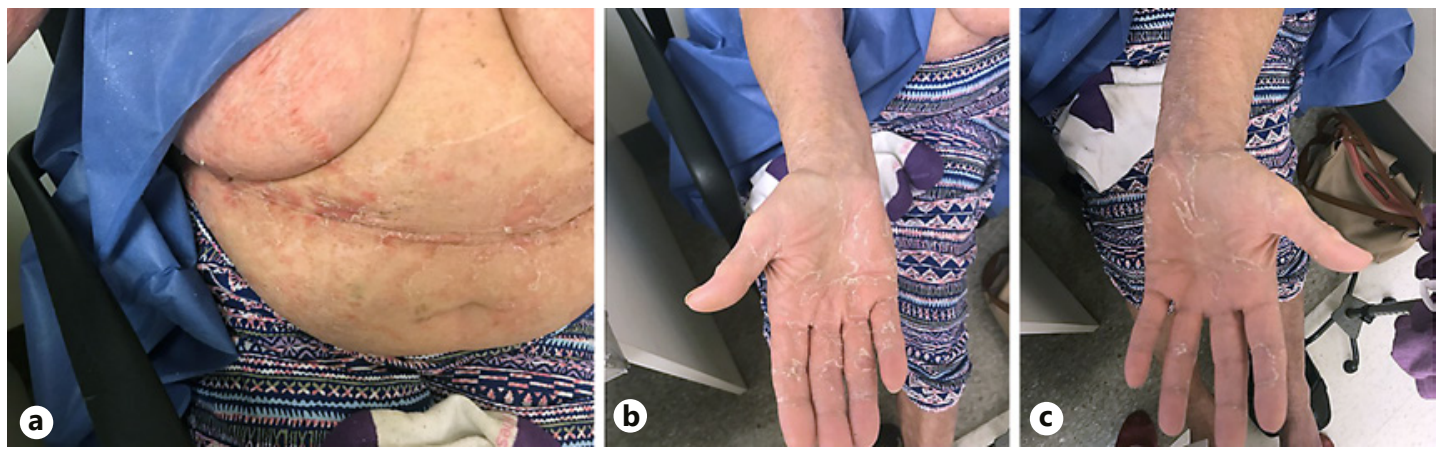

Fig. 1. Clinical Images from initial presentation. a Gyrate erythema with desquamating scale on the abdomen and right breast. b, c Bilateral palmar desquamation.
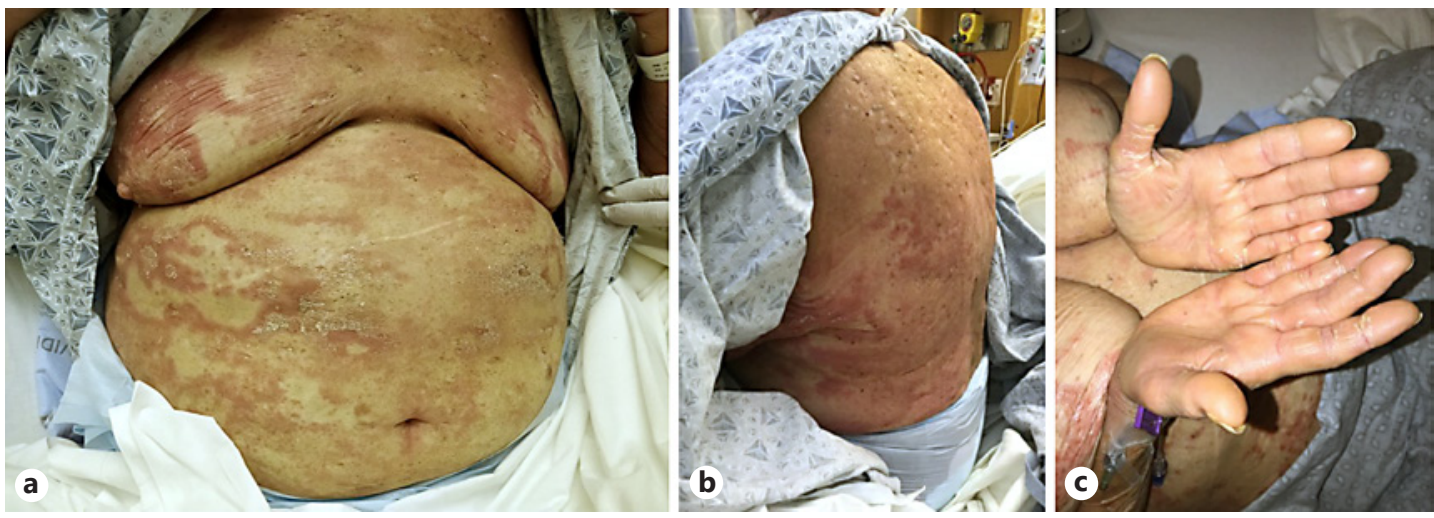

Fig. 2. Clinical images 6 weeks after initial presentation. a, b On the chest, abdomen, and back, annular erythematous patches and plaques with occasional central clearing and overlying scale are present. c Bilateral palmar desquamation and keratoderma.

The rash was associated with significant pruritus. The patient felt otherwise well, and her psoriasis and hidradenitis suppurativa were well controlled with acitretin. Her past medical history was notable for hypertension, for which she had started lisinopril and amlodipine several months prior to the onset of her rash. The patient was up-to-date on age-appropriate malignancy screening. A skin biopsy obtained at the initial dermatology visit was consistent with erythema annulare centrifugum (EAC). Given the concern for a drug-induced etiology, the patient's aforementioned antihypertensive medications were discontinued, and she was transitioned from metoprolol to clonidine.

Over the following 6 weeks, the patient's rash significantly worsened, spreading to her back and lower extremities, and she developed bilateral palmar keratoderma (Fig. 2). As the etiology of her presumed EAC remained unidentified, the patient underwent an extensive malignancy evaluation - including complete blood count, serum protein electrophoresis, computed tomography scans of her chest, abdomen, and pelvis, and urine cytology - which was unremarkable. After self-discontinuing clonidine, the patient presented to the dermatology clinic, where she reported worsening blurry vision and was noted to have a systolic blood pressure of $210 \mathrm{~mm} \mathrm{Hg}$, for which she was admitted to the hospital. 

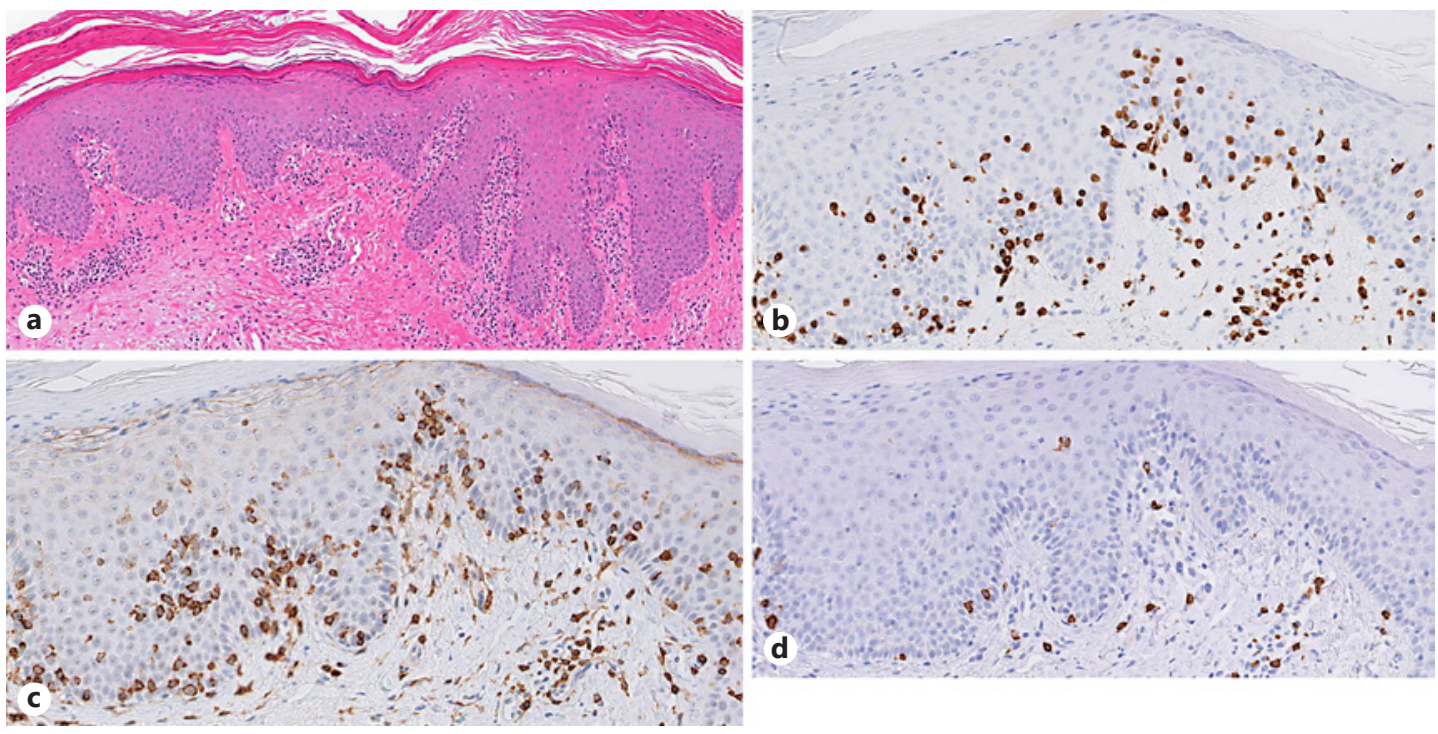

Fig. 3. Histopathological images of the case. Punch biopsy of the right upper extremity. a Hematoxylin and eosin stain shows an atypical lymphocytic infiltrate with epidermotropism. b A CD3 immunohistochemical stain reveals that the atypical lymphocytes are $\mathrm{T}$ cells. c, d Immunostaining demonstrates an abnormal CD4:CD8 ratio, with CD4+ T cells predominating.

\section{Diagnosis and Clinical Course}

Upon admission, the patient was restarted on metoprolol and clonidine given she had progression of her disease despite a trial off of these medications. Dermatology was consulted. On examination, she had annular plaques with scattered firm pink papules, worsening hyperkeratosis and fissuring of her palms as well as severe pruritus. Given the patient's disease progression, lack of an identifiable trigger for EAC, and increased clinical concern for cutaneous T-cell lymphoma (CTCL), a repeat skin biopsy was performed during her admission. Histopathologic evaluation revealed an atypical lymphocytic infiltrate with epidermal tagging and cytological atypia manifested as large cells with irregular nuclear contours (Fig. 3a). Immunohistochemistry demonstrated that the infiltrate consisted of predominantly CD3+ T cells (Fig. 3b), with an increased CD4:CD8 ratio of approximately 5:1 (Fig. 3c, d). T-cell receptor- $\gamma$ (TCR) gene rearrangement studies performed on both the prior biopsy as well as the current biopsy revealed a similar clone shared by both biopsies. Taken together, the findings support a diagnosis of mycosis fungoides (MF). Flow cytometry and TCR gene rearrangement studies performed on peripheral blood did not show evidence of systemic involvement, i.e., Sézary syndrome.

These immunophenotypic findings, in conjunction with the patient's rash involving approximately $40 \%$ of her body surface area, yielded the final diagnosis of stage IB (T2N0M0) MF. The patient was ultimately treated with high-potency topical corticosteroids and topical calcipotriene, which provided rapid and sustained disease control.

\section{Discussion}

MF, the most common CTCL [1], is characterized in its early stage by erythematous patches and plaques with overlying fine scale and an anatomic predisposition for sunprotected areas [2]. Histopathologically, MF is characterized by a clonal proliferation of 
Molina et al.: A 78-Year-Old Female with a Diffuse Pruritic Rash and Palmoplantar Desquamation

atypical, epidermotropic T cells [3]. In addition to the well-known clinicopathological heterogeneity of its various presentations [4, 5], MF has been reported to imitate numerous inflammatory and noninflammatory dermatoses [6,7]. Among these multiform morphologic presentations of MF, EAC-like lesions are particularly rare and scarcely reported in the literature [8-11].

EAC is a chronic, reactive dermatosis that is considered a delayed-type hypersensitivity response to a wide variety of potential antigens [12]. Known triggers include malignancy, medications, cutaneous fungal infections, and various autoimmune diseases [13]. EAC is characterized by annular erythematous plaques that gradually extend peripherally, resulting in centrally clearing and trailing scale [12]. Histopathological examination differentiates superficial from deep EAC depending on the extent of perivascular lymphocytic infiltrate, and deep EAC tends to present with an infiltrated border and minimal scale $[14,15]$.

Our report offers an unusual case of a patient with MF, whose lesions initially appeared clinically and histologically consistent with EAC. Among the reported EAC-like MF cases in the literature, even lesions with a classic EAC appearance all demonstrated histopathologic features suggestive of MF [8-11]. In contrast, our patient's initial skin biopsy revealed a dense superficial dermal perivascular lymphocytic infiltrate with spongiosis and parakeratosis, more suggestive of EAC rather than MF $[14,15]$.

However, our patient's clinical course exhibited several features that were inconsistent with EAC. Palmoplantar keratoderma, for instance, is unusual in EAC but can be seen in MF $[4,16,17]$. Similarly, intractable pruritus - which was prominent in our patient - would be an atypical feature of EAC but is characteristic of MF lesions [18-20]. After a thorough investigation and discontinuation of potential offending medications failed to identify a trigger for our patient's presumed EAC, the diagnosis of MF was made when a repeat skin biopsy revealed abnormal, epidermotropic $\mathrm{T}$ cells. In addition to standard histopathological examination, immunophenotyping studies can help distinguish CTCL from inflammatory or reactive lymphoid infiltrates. For example, the absence of mature T-cell markers - i.e., CD2, CD5, and CD7 [21] - and the presence of a clonal T-cell population [22] are both suggestive of CTCL. Furthermore, certain diagnostic features - such as erythroderma, matching clonal TCR rearrangement in the blood, and specific immunophenotypic T-cell findings - distinguish MF from Sézary syndrome, an aggressive leukemic variant of CTCL [23].

Staging for CTCL is based on the morphological appearance of the cutaneous lesions (patches, plaques, tumors), the body surface area involved, lymphadenopathy, and involvement of blood or visceral organs [23]. Our patient presented with patches and plaques covering $40 \%$ of her body surface area but without lymphadenopathy or blood abnormalities, corresponding to stage IB (T2N0M0) disease. Treatment is guided by the disease stage, whereby early-stage MF is generally managed with skin-directed therapies (topical corticosteroids, topical retinoids, phototherapy) and late-stage MF may require extracorporeal photophoresis, radiation, and systemic agents including methotrexate or chemotherapy [18].

Unfortunately, our patient self-discontinued necessary antihypertensive medications under the belief that her skin disease was drug induced. Once the diagnosis of MF was eventually realized, the patient was initiated on appropriate topical therapy and experienced near complete resolution of her skin lesions and associated pruritus. She was furthermore able to restart her antihypertensive medications without the fear of a potential flare in her rash. Our case serves as a reminder not only of the impressive array of dermatoses that MF can simulate, but also of the importance of clinicopathological correlation in the diagnosis of MF, and the need to reconsider one's initial diagnosis especially when patients demonstrate clinical presentations that seem inconsistent with their histopathological diagnoses. 
Molina et al.: A 78-Year-Old Female with a Diffuse Pruritic Rash and Palmoplanta Desquamation

\section{Statement of Ethics}

This article was prepared in compliance with all ethical and confidentiality guidelines and principles. The patient has provided her written informed consent.

\section{Disclosure Statement}

The authors have no conflicts of interest to disclose.

\section{References}

1 Bradford PT, Devesa SS, Anderson WF, Toro JR. Cutaneous lymphoma incidence patterns in the United States: a population-based study of 3884 cases. Blood. 2009 May;113(21):5064-73.

2 Pimpinelli N, Olsen EA, Santucci M, Vonderheid E, Haeffner AC, Stevens S, et al.; International Society for Cutaneous Lymphoma. Defining early mycosis fungoides. J Am Acad Dermatol. 2005 Dec;53(6):1053-63.

3 Smoller BR, Bishop K, Glusac E, Kim YH, Hendrickson M. Reassessment of histologic parameters in the diagnosis of mycosis fungoides. Am J Surg Pathol. 1995 Dec;19(12):1423-30.

4 Kazakov DV, Burg G, Kempf W. Clinicopathological spectrum of mycosis fungoides. J Eur Acad Dermatol Venereol. 2004 Jul;18(4):397-415.

5 Muñoz-González H, Molina-Ruiz AM, Requena L. Clinicopathologic Variants of Mycosis Fungoides. Actas Dermosifiliogr. 2017 Apr;108(3):192-208.

6 Nashan D, Faulhaber D, Ständer S, Luger TA, Stadler R. Mycosis fungoides: a dermatological masquerader. $\mathrm{Br}$ J Dermatol. 2007 Jan;156(1):1-10.

7 Zackheim HS, McCalmont TH. Mycosis fungoides: the great imitator. J Am Acad Dermatol. 2002 Dec;47(6): 914-8.

8 Moura FN, Thomas L, Balme B, Dalle S. Mycosis fungoides mimicking an annular erythema. Clin Exp Dermatol. 2009 Dec;34(8):e581-3.

9 Ceyhan AM, Akkaya VB, Chen W, Bircan S. Erythema annulare centrifugum-like mycosis fungoides. Australas J Dermatol. 2011 Nov;52(4):e11-3.

10 Imafuku K, Nomura Y, Nakayama C, Abe R, Endo T, Shimizu H. Erythema annulare centrifugum-like mycosis fungoides after unrelated bone marrow transplantation. Br J Haematol. 2015 Jul;170(2):140.

11 Saada D, Lami MC, Vabres P, Levillain P, Guillet G. [Mycosis fungoides presenting as annular erythema]. Ann Dermatol Venereol. 2005 Jan;132(1):35-7.

12 Goldsmith LA, Katz SI, Gilchrest BA, Paller AS, Leffell DJ, Wolff K. Fitzpatrick's dermatology in general medicine. 8th ed. New York: McGraw-Hill; 2012. pp. 463-6.

13 Kim DH, Lee JH, Lee JY, Park YM. Erythema annulare centrifugum: analysis of associated diseases and clinical outcomes according to histopathologic classification. Ann Dermatol. 2016 Apr;28(2):257-9.

14 Kim KJ, Chang SE, Choi JH, Sung KJ, Moon KC, Koh JK. Clinicopathologic analysis of 66 cases of erythema annulare centrifugum. J Dermatol. 2002 Feb;29(2):61-7.

15 Weyers W, Diaz-Cascajo C, Weyers I. Erythema annulare centrifugum: results of a clinicopathologic study of 73 patients. Am J Dermatopathol. 2003 Dec;25(6):451-62.

16 Aram H, Zeidenbaum M. Palmoplantar hyperkeratosis in mycosis fungoides. J Am Acad Dermatol. 1985 Nov; 13(5 Pt 2):897-9.

17 Topf S, Lüftl M, Neisius U, Brabletz T, Simon M Jr, Schuler G, et al. Mycosis fungoides palmaris et plantaris-an unusual variant of cutaneous T-cell lymphoma. Eur J Dermatol. 2006 Jan-Feb;16(1):84-6.

18 Al Hothali GI. Review of the treatment of mycosis fungoides and Sézary syndrome: A stage-based approach. Int J Health Sci (Qassim). 2013 Jun;7(2):220-39.

19 Ahern K, Gilmore ES, Poligone B. Pruritus in cutaneous T-cell lymphoma: a review. J Am Acad Dermatol. 2012 Oct;67(4):760-8.

20 Vij A, Duvic M. Prevalence and severity of pruritus in cutaneous T cell lymphoma. Int J Dermatol. 2012 Aug; 51(8):930-4.

21 Smoller BR, Santucci M, Wood GS, Whittaker SJ. Histopathology and genetics of cutaneous T-cell lymphoma. Hematol Oncol Clin North Am. 2003 Dec;17(6):1277-311.

22 Ashton-Key M, Diss TC, Du MQ, Kirkham N, Wotherspoon A, Isaacson PG. The value of the polymerase chain reaction in the diagnosis of cutaneous T-cell infiltrates. Am J Surg Pathol. 1997 Jul;21(7):743-7.

23 Olsen E, Vonderheid E, Pimpinelli N, Willemze R, Kim Y, Knobler R, et al.; ISCL/EORTC. Revisions to the staging and classification of mycosis fungoides and Sezary syndrome: a proposal of the International Society for Cutaneous Lymphomas (ISCL) and the cutaneous lymphoma task force of the European Organization of Research and Treatment of Cancer (EORTC). Blood. 2007 Sep;110(6):1713-22. 